\title{
Discovery of Azurin-Like Anticancer Bacteriocins from Human Gut Microbiome through Homology Modeling and Molecular Docking against the Tumor Suppressor p53
}

\author{
Chuong Nguyen ${ }^{1,2}$ and Van Duy Nguyen ${ }^{3}$ \\ ${ }^{1}$ Theoretical Physics Research Group, Ton Duc Thang University, Ho Chi Minh City, Vietnam \\ ${ }^{2}$ Faculty of Applied Sciences, Ton Duc Thang University, Ho Chi Minh City, Vietnam \\ ${ }^{3}$ Institute of Biotechnology and Environment, Nha Trang University, Nha Trang, Khanh Hoa, Vietnam \\ Correspondence should be addressed to Van Duy Nguyen; duynv@ntu.edu.vn
}

Received 26 November 2015; Revised 31 March 2016; Accepted 12 April 2016

Academic Editor: Jose C. Valverde

Copyright (C) 2016 C. Nguyen and V. D. Nguyen. This is an open access article distributed under the Creative Commons Attribution License, which permits unrestricted use, distribution, and reproduction in any medium, provided the original work is properly cited.

\begin{abstract}
Azurin from Pseudomonas aeruginosa is known anticancer bacteriocin, which can specifically penetrate human cancer cells and induce apoptosis. We hypothesized that pathogenic and commensal bacteria with long term residence in human body can produce azurin-like bacteriocins as a weapon against the invasion of cancers. In our previous work, putative bacteriocins have been screened from complete genomes of 66 dominant bacteria species in human gut microbiota and subsequently characterized by subjecting them as functional annotation algorithms with azurin as control. We have qualitatively predicted 14 putative bacteriocins that possessed functional properties very similar to those of azurin. In this work, we perform a number of quantitative and structurebased analyses including hydrophobic percentage calculation, structural modeling, and molecular docking study of bacteriocins of interest against protein p53, a cancer target. Finally, we have identified 8 putative bacteriocins that bind p53 in a same manner as p28-azurin and azurin, in which 3 peptides (p1seq16, p2seq20, and p3seq24) shared with our previous study and 5 novel ones (p1seq09, p2seq05, p2seq08, p3seq02, and p3seq17) discovered in the first time. These bacteriocins are suggested for further in vitro tests in different neoplastic line cells.
\end{abstract}

\section{Introduction}

As one of the most deadly diseases worldwide, cancer is involved in disregulation of mammalian cell differentiation and growth. There is now no conceivable way that current drugs can prevent cancer relapse once the cancer is in remission. The common treatment of cancer is undertaking surgical resection of the tumors followed by radiation and chemotherapy [1]. There are two types of drugs that are normally used in chemotherapy, including small molecule drugs (e.g., tyrosine kinase inhibitors) and human or humanized proteins (e.g., monoclonal antibodies). However, these "one drug-one target" therapies can cause the most devastating side effects on the growth of normal cells and lead to the rapid resistance to drugs developed by the cancer cells using alternate pathways for growth or using efflux pumps to pump out drugs [2]. Therefore, new therapies for cancer drug discovery using multitargeted approaches to overcome resistance, toxicity, and side effects are urgently needed.

Over the past centuries, a phenomenon of spontaneous regression of tumors associated with bacterial infections has been observed [3]. One of the most well-known treatments based on this phenomenon was reported in late 1890s by an American physician, Coley [4]. He observed the relationship between bacterial infection and cancer regression, which led to the discovery of a killed bacterial vaccine for cancer, known as "Coley's toxin" [3]. This suggested renewed interest in the development of new therapeutic anticancer modalities based on the use of live bacteria and their purified products including bacterial toxins, proteins, peptides, and enzymes. Recently, a number of bacterial proteins and peptides have been described to exert an anticancer activity at preclinical level toward diverse types of cancer cells [1]. Among them, bacteriocins are antimicrobial peptides or proteins 
ribosomally synthesized by bacteria to inhibit the growth of the similarly or closely related bacterial strains (narrow spectrum) and sometimes against a wide spectrum of species. They have been looking for a positive health benefit to the host including human, livestock, aquaculture animals, and some plants [5]. Bacteriocins promise to be effective therapeutic agent and their biochemical properties have been studied; their antineoplastic capability has also identified after its discovery in the late 1970s by using crude bacteriocin preparation toxic to mammalian cells [6]. Common bacteriocins like pyocin, colicin, pediocin, and microcin have been shown to possess inhibitory properties against different neoplastic line cells [5].

Among well-known protein anticancer agents in bacteria, there are immunotoxins and several bacterial proteins including Mycobacterium bovis MPT63, arginine deiminase from Mycoplasma arginini, lipidated azurin (laz) from Neisseria meningitides, and azurin from Pseudomonas aeruginosa [1]. The latest one, azurin, is an important bacteriocin, a member of the cupredoxin family of redox proteins, which becomes a potential anticancer drug because of some of its unique properties. Azurin can preferentially penetrate human cancer cells and exerts cytostatic and apoptotic effects with no apparent activity on normal cells [7, 8]. Azurin can directly interact and stabilize the tumor suppressor p53 [7]. The azurin domain responsible for its specific entry in cancer cells was demonstrated that it spans residues 50-77 (termed p28) and adopts an amphipathic alpha helical conformation [9]. Cell penetration is not accompanied by membrane disruption, which could cause cell death. Preclinical evaluation of pharmacokinetics, metabolism, and toxicity of azurin-p28 was evaluated [10], establishing it as nonimmunogenic and nontoxic in mice and nonhuman primates. Moreover, the protein-protein interactions between azurin and p53 have recently been analyzed by bioinformatics and atomic force microscopy [11-13].

Interestingly, not only does azurin have anticancer activity but also it strongly inhibits host cell invasion by the AIDS virus HIV-1 [14], the malarial parasite Plasmodium falciparum [14], and the toxoplasmosis-causing parasite Toxoplasma gondii [15]. Thus azurin is believed to be a weapon used by $P$. aeruginosa to keep invaders of the human body for long term residence without harming or exerting any toxicity to the host [1]. This also suggests that azurin may be specific for tumors in the organs where $P$. aeruginosa normally resides during infection. In fact, Neisseria meningitides produces an azurin-like protein called laz (lipidated azurin) with a 127 amino acid moiety with $56 \%$ amino acid sequence identity to $P$. aeruginosa azurin. Several US patents have been issued to cover the use of azurin and laz in cancer therapies [16], and azurin has shown significant activity, as well as enhancement of the activity of other drugs, in oral squamous carcinoma cells [17].

The very important question is whether azurin is the only bacteriocin produced by $P$. aeruginosa as an anticancer weapon or whether there are other bacteriocins, produced by other bacteria with the ability to cause chronic infections and have long term residence in human bodies, as well as defending the body from invaders such as viruses and parasites. It is, thus, interesting to note that azurin is not the only anticancer bacteriocins produced by human microflora. In fact, their antineoplastic properties have been inadequately revealed in the late 1970 s by using crude bacteriocin preparation toxic to mammalian cells. Nowadays, purified bacteriocins are available and have shown inhibitory properties toward diverse neoplastic line cells. Pyocin, colicin, pediocin, and microcin are among bacteriocins reported to present such activity $[5,18]$.

Although bacteriocins have been found in many major lineages of bacteria and some members of the Archaea, more and more new bacteriocins with new characteristics and origins are still awaiting discovery. By now, bacteriocins have mainly been derived from the lactic acid bacteria with mostly fermented food origins. Besides, colicins from E. coli were used as model Gram-negative bacteriocins. There were only a few basic researches on noncolicin bacteriocins of human origins and bacteriocins with killing activity against eukaryotic and human cells [5].

Here, we hypothesize thatbacteria from human microflora, especially pathogenic and commensal bacteria, with long term residence in human body can produce azurinlike bacteriocins as a weapon to protect their habitat from cancers. In our previous work [19], putative bacteriocins have been screened from complete genomes of 66 dominant bacteria species in human gut microbiome and subsequently characterized by subjecting them as functional annotation algorithms with azurin as control. We have predicted a number of bacteriocins possessed functional properties very similar to those of azurin [19]. However, the study was limited to qualitative assessment of the bacteriocins at sequencelevel only. In addition, the hydrophobicity of the peptides, which is suggested to play an important role on anticancer activity, has not been addressed. Therefore, in this study, we performed a system of quantitative analyses including functional prediction (using a scoring function to evaluate) and hydrophobic percentage calculation to identify azurinlike bacteriocins. Next, to extend our analysis at structural level, we performed structural modeling and molecular docking study of bacteriocins of interest against protein p53, a cancer target. These analyses provided us more reliable data to identify azurin-like bacteriocins with potential anticancer activity.

\section{Materials and Methods}

2.1. Screening of Potential Bacteriocin Sequences from Human Gut Microbiome. In our previous work, hypothetical bacteriocins which possess properties similar to azurin have been suggested [19]. In summary, the complete genomes of 66 dominant species among 101 prevalent gut microbial species [20] were retrieved from the National Center for Biotechnology Information (NCBI) Databases (http://ncbi.nlm.nih.gov/). They were then scanned using the BAGEL web server (http://bagel.molgenrug.nl/) [21] in order to identify putative genes encoding bacteriocins. The output of BAGEL is the protein sequences of hypothetical bacteriocins. The sequences were saved in FASTA format for subsequent analysis (Figure 1). 


\begin{tabular}{|c|c|c|c|c|c|}
\hline $\begin{array}{l}\text { Screening } \\
\text { bacteriocins } \\
\text { from gut } \\
\text { microbial } \\
\text { species using } \\
\text { BAGEL server }\end{array}$ & $\begin{array}{l}\text { Screening } \\
\text { azurin-like } \\
\text { bacteriocins } \\
\text { using } \\
\text { functional } \\
\text { prediction } \\
\text { ProtFun } \\
\text { server }\end{array}$ & $\begin{array}{l}\text { Calculation of } \\
\text { hydrophobic } \\
\text { percentage of } \\
\text { chosen } \\
\text { bacteriocins }\end{array}$ & $\begin{array}{l}\text { Structural } \\
\text { prediction of } \\
\text { chosen } \\
\text { bacteriocins } \\
\text { using I- } \\
\text { TASSER } \\
\text { server }\end{array}$ & $\begin{array}{l}\text { Structural } \\
\text { evaluation } \\
\text { and } \\
\text { refinement } \\
\text { using } \\
\text { Molproblity, } \\
\text { GalaxyWEB, }\end{array}$ & $\begin{array}{l}\text { Protein-protein } \\
\text { docking of the } \\
\text { chosen } \\
\text { bacteriocins } \\
\text { models against } \\
\text { p53 DBD using } \\
\text { Cluspro server }\end{array}$ \\
\hline
\end{tabular}

FIGURE 1: Flowchart illustrating the process of identification and subsequent characterization of hypothetical anticancer bacteriocins from gut microbial species.

2.2. Screening Azurin-Like Bacteriocins Using Functional Prediction ProtFun Server. The hypothetical bacteriocins sequences were subjected to the web server ProtFun 2.2 (http://www.cbs.dtu.dk/services/ProtFun-2.2/) [22, 23]. The ProtFun 2.2 server produces $a b$ initio predictions of protein function from sequence. The $a b$ initio feature is important for hypothetical bacteriocins because most of the sequences predicted from BAGEL server in previous step are not yet available in any sequence database. For each input sequence, the server predicted cellular function (F), enzyme class (EZ), and gene ontology (GO) category. The scores of the prediction consist of two numbers: the probability and the odds number. The first number is the estimated probability that the entry belongs to the class in question, being influenced by the prior probability of that class. The second number represents the odds that the given sequence belongs to that class/category. It is independent of the prior probability.

In this work, we use the odds number to rank our hypothetical bacteriocins using azurin as a control. To estimate the similarity between each hypothetical bacteriocin sequence and azurin, we collect the predicted odds number that the sequence belongs to the same category with azurin. The score of similarity of a sequence with azurin is estimated using logarithm of production of three odds numbers:

$$
\begin{aligned}
& \text { Score }(\text { seq })=\log [\text { odds }(\text { seq } \mid \text { azurin_F }) \\
& \times \text { odds }(\text { seq | azurin_EZ) } \\
& \times \text { odds (seq | azurin_GO) }] .
\end{aligned}
$$

The higher the score, the higher the odds that the sequence belongs to the same categories with the azurin. All the sequences with the score higher than 0 were selected for further analysis.

2.3. Calculation of Hydrophobic Percentage. Hydrophobic interaction is relatively stronger than other weak intermolecular forces such as Van der Waals interactions or hydrogen bonds and is suggested to play an important role for membrane permeabilization and in antitumor activity [24]. Therefore, information about hydrophobic amino acids in short peptides is considered to be among the key criteria for ranking.

The sequences from previous step were subjected to calculations of hydrophobic percentage. In other words, these calculations give us the percentage of hydrophobic amino acids with respect to the total amino acids of the sequence. The residues considered as hydrophobic are Phenylalanine
(Phe, F), Isoleucine (Ile, I), Leucine (Leu, L), Methionine (Met, M), Valine (Val, V), Tryptophan (Try, W), Glycine (Gly, G), Cystein (Cys, C), and Alanine (Ala, A). All hypothetical bacteriocins as well as azurin and p28-azurin were subjected to hydrophobic percentage calculation. Those bacteriocins which have hydrophobic percentage similar to azurin are considered to be promising azurin-like anticancer bacteriocins and selected for next step.

2.4. Protein Structure Prediction. Because 3D structures of all the bacteriocins selected from previous step have not been available in RCSB Protein Data Bank yet; modeling of these peptides was done by using I-TASSER server (http://zhanglab.ccmb.med.umich.edu/I-TASSER). ITASSER is a hierarchical bioinformatics method for predicting three-dimensional structure of protein molecules from amino acid sequences [25]. This method detects template structures from the Protein Data Bank (PDB) by multiple threading approaches. The full-length atomic models are constructed by iterative template fragment assembly simulations using replica exchange Monte Carlo method. I-TASSER (as previously called "Zhang-Server") is ranked as the best server for protein structure prediction in recent Critical Assessment of Structure Prediction (CASP) experiments [25].

All chosen sequences (in FASTA format) were submitted to I-TASSER server. For each sequence, the server predicted a number of models and ranked them by $C$-score. The $C$ score is typically in the range of $(-5,2)$, where a $C$-score of higher value signifies a model with a high confidence. The model with the highest $C$-score was selected and refined by GalaxyWEB [26] and ModRefiner [27] servers. The models before and after refinement were all validated for their backbone conformation geometry and the residue contact using MolProbity [28].

\subsection{Docking Potential Anticancer Bacteriocins against Cancer} Target $p 53$. Protein-protein docking approach was used to predict binding poses of the potential anticancer bacteriocins selected from the previous step to the common cancer target $\mathrm{p} 53$ DNA binding domain. The anticancer $\mathrm{p} 28$ peptide fragment of azurin was also docked against p53 to validate the docking method. The protein p53 core domain mutant (PDB ID 2J1X) obtained from Protein Data Bank [29] was chosen as the receptor for docking. Cluspro 2.0 [30], a fully automated web-based program for the computational docking of protein structures, was employed. Cluspro 2.0 proteinprotein docking algorithm works in three main steps. In first step, it runs PIPER, based on a Fast Fourier Transform (FFT) 
docking method. Secondly, it used a clustering approach for the identification of near native conformations and discards the unstable clusters. Finally, a short Monte Carlo simulation was applied to judge the stability of these clusters and further refined [30]. For each peptide, the best model based on cluster size was chosen for further analysis.

\section{Results and Discussion}

3.1. Screening Azurin-Like Bacteriocins Using Functional Prediction ProtFun Server. Prediction of functions of azurin from Pseudomonas aeruginosa (uniprot ID: P00282) and all 81 putative bacteriocins using ProtFun server is calculated and shown in Table S1 (Supplementary Material available online at http://dx.doi.org/10.1155/2016/8490482). Azurin is predicted as cellular envelope (odds 9.71), nonenzyme (odds 1.147), and gene oncology of immune response (odds 5.877). In this work, the three functional categories are considered equally important. Thus, the score of similarity in (1) is the result of production of three odd numbers without any weighted coefficient for each odd number (odds product 65.5). This score helps us avoid biasing toward any particular property of the sequence.

The sequences of putative bacteriocins are ranked by odd score from high to low. The higher the score, the higher the odds that the sequence belongs to the same categories with the azurin. There are 28 sequences with odds score greater than 0 . They are considered most similar to azurin among all 81 putative bacteriocins. Considering each odd score, $\mathrm{p} 3 \mathrm{seq} 02$ has largest immune response odds at 5.0, which is smaller than that of azurin. However, p1seq04 has largest nonenzyme odds (1.2) among others, while p2seq07 has the largest cellular envelope odds (10.2) being the top hit of the odds product at 25.5. Although p3seq02 has the largest immune response odds compared to the p2seq07 (4.1), it has much lower nonenzyme odds and cellular envelope odds compared to p2seq07 and azurin.

The probability score for each sequence was also calculated. We found that there is a strong correlation between the odds score and probability score with correlation coefficient $R=0.998$ (Figure S1, Supplementary Material). This result suggests that the probability score can also be used as odds score to rank the similarity of bacteriocins and azurin.

Thus, in this first step we identified 28 novel putative bacteriocins possessed functional properties very similar to that of azurin. ProtFun results have predicted that azurin has cell envelope function while our bacteriocins expressed the same properties, revealing a great chance of possessing cancer cell attack activity similar to that of azurin. The more important functional characterization, which was to predict the propensity of the bacteriocins, was enzymatic activity. The low propensity of azurin to have enzymatic activity indicates that it has little chance of interacting with any other substrates or altering the normal cellular kinetics. This is consistent with the special feature of azurin which targets human cancer cells without exerting activity on normal cells [7-9]. Like azurin, our bacteriocins showed immune responses which have very low chances of drug-induced adverse reactions of type B which comprise idiosyncratic and immune-mediated side effects [31]. These results signify that our selected bacteriocins have great chance of having anticancer activity similar to that of azurin.

3.2. Calculation of Hydrophobic Percentage. It has been suggested that the hydrophobicity of a peptide plays a crucial role in the mechanism of action against cancer cells, which should be taken into account in the design of potential anticancer peptides $[32,33]$. The anticancer activity of a peptide has been shown to be correlated with the peptide hydrophobicity which means that increasing hydrophobicity leads to the increase of anticancer activity [33]. Peptides with higher hydrophobicity are suggested to enter deeper into the hydrophobic core of the cell membrane, causing stronger activity of disrupting the cancer cell membrane [33]. On the other hand, the peptide specificity against cancer cells and normal cells depends on the hydrophobicity in different manner. Too low or too high hydrophobicity reduces the specificity of the peptide against cancer cells [33]. Thus, a designed peptide should have the hydrophobicity in the range that has the capability of entering cells as well as the specificity against cancer cells.

One common index that can be used to measure the hydrophobicity is the percentage of hydrophobic residues in each sequence. The hydrophobic percent of azurin sequence and p28-azurin (amino acids 50 to 77 of azurin) is $49 \%$ and $46 \%$, respectively. Azurin and p28-azurin have been shown to have entry specificity in cancer cells and prevent cancer cell growth by interfering in multiple pathways by which cancer cells grow [34]. Interestingly, p18-azurin (amino acids 50 to 67 of azurin) has been shown to be responsible for the entry of azurin into human cancer cells but not for the inhibition of cancer cell proliferation [35]. The hydrophobic percent of p18-azurin is $61 \%$ that is much higher than the hydrophobic percents of azurin and p28-azurin. Thus, to screen out putative bacteriocins with anticancer potential as azurin, the sequences that have hydrophobic percentage from $44 \%$ to $51 \%$ are selected. This corresponds to the fact that hydrophobic percentage of chosen bacteriocins is within $2 \%$ deviation from that of azurin or p28-azurin.

Among 28 putative azurin-like bacteriocins, there are 14 sequences that satisfy these criteria (Table 1). In which, 10 of them are small peptides (7 Sactipeptides, 2 Lasso peptides, and 1 UviB class II) and 4 of them are large proteins (all Zoocin A type). These sequences are found in 7 bacterial species including Anaerotruncus colihominis (1 sequence), Bacteroides vulgatus (2 sequences), Clostridium hathewayi (reclassified as Hungatella hathewayi) (1 sequence), Clostridium nexile (6 sequences), Dorea longicatena (1 sequence), Eubacterium ventriosum (1 sequence), and Ruminococcus sp. (2 sequences). Only Bacteroides vulgatus is a species within the Order Bacteroidales, whereas all remaining species belong to the Order Clostridiales. A molecular genetic analysis of rDNA amplicons generated directly from a human faecal sample showed that more than $90 \%$ of the flora could be assigned to three major phylogenetic lineages (the Bacteroides, Clostridium coccoides, and Clostridium leptum groups) [36]. Thus our bacteriocins were produced by the most dominant bacterial species in human gut. Interestingly, 


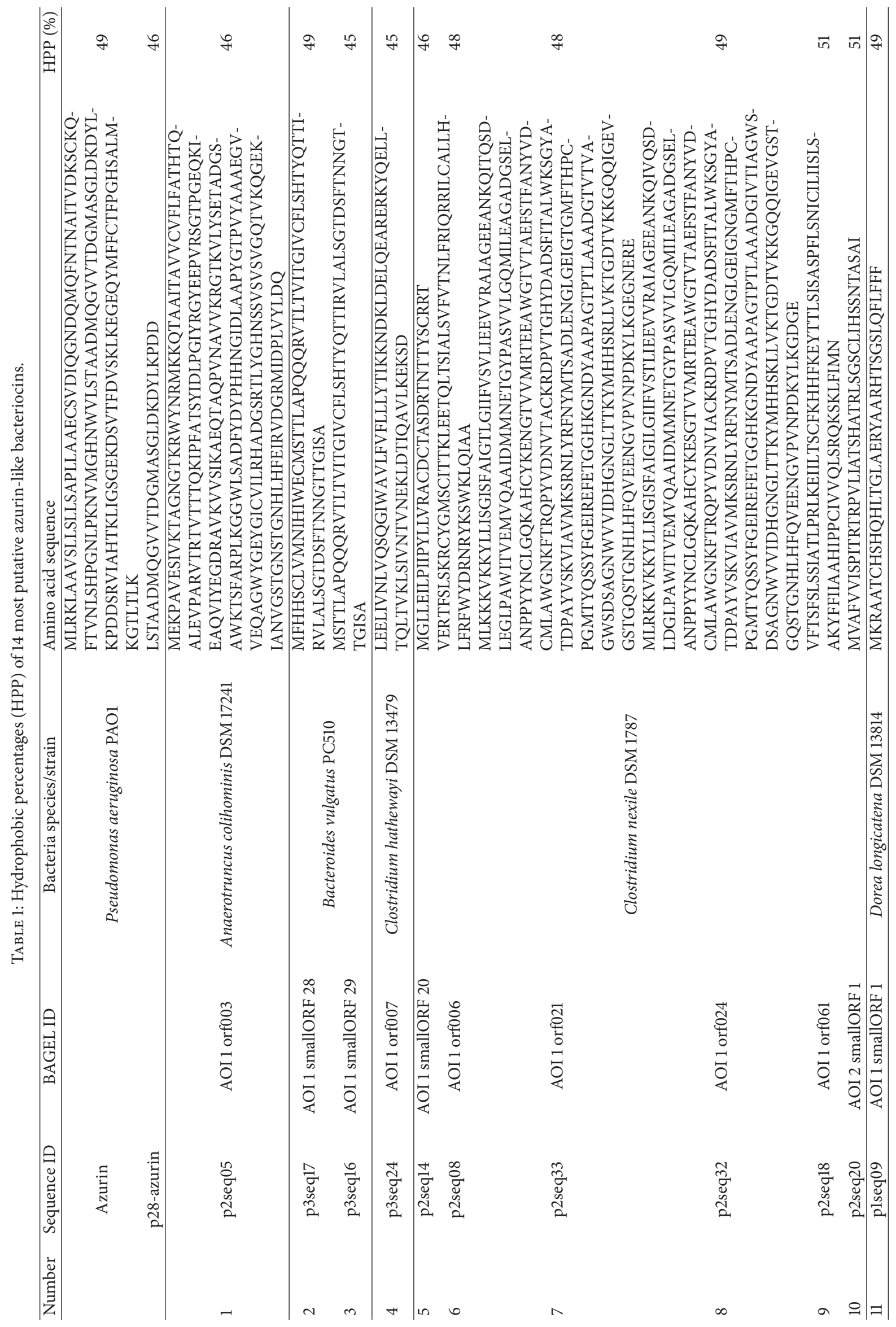




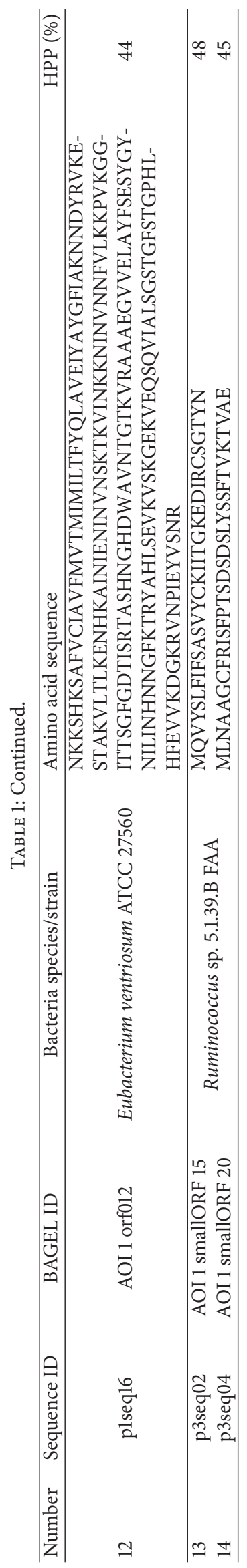




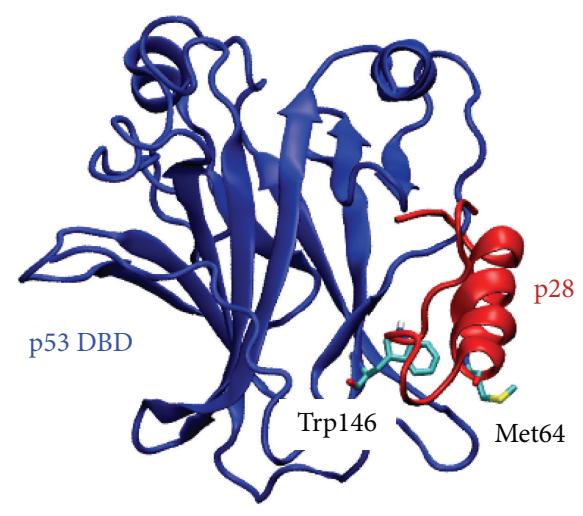

(a)

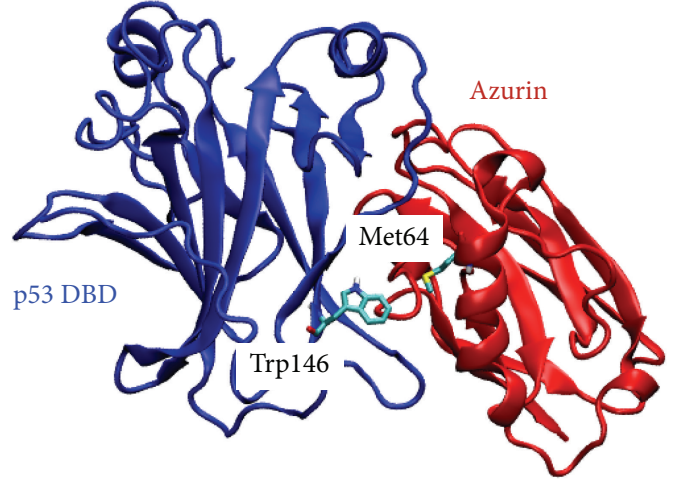

(b)

FIgURE 2: Three-dimensional configurations of docking models of p53 DBD with p28-azurin (a) and with azurin (b).

most of putative azurin-like bacteriocin-producing species are considered as pathogenic bacteria, which fits well with our hypothesis. The results are also in agreement with cases of azurin-producing Pseudomonas aeruginosa and lazproducing Neisseria meningitides.

3.3. Structural Prediction of Chosen Bacteriocins. I-TASSER is one of the most popular online servers for automated protein structure prediction. The accuracy of generated structural models of I-TASSER is comparable to the best human-expert guide modeling [25]. This server can be used for sequences with or without similar folds in the Protein Data Bank (PDB) library [37]. Thus, tertiary structures of 14 putative bacteriocin sequences were predicted using ITASSER server [25] and then refined by GalaxyWEB [26] and ModRefiner [27] servers. Predicted structures are shown in Figure S2 (Supplementary Material). The percentage of ramachandran favored residues and rotamer favored residues of 14 refined models is $90.2 \%$ and $97.4 \%$ on average (Table S2, Supplementary Material).

These data also showed that, for each bacteriocin, the quality of the model improves significantly after refinement. The MolProbity scores of all refined models were also calculated. MolProbity score combines the clash score, rotamer, and Ramachandran evaluations into a single score, normalized to be on the same scale as X-ray resolution [28]. The scores of all refined models are below 1.5 indicating that our models have the quality of corresponding X-ray resolution [28].

A new database of structurally annotated therapeutic peptides, SATPdb (http://crdd.osdd.net/raghava/satpdb/), which is curated from 22 public domain peptide databases/ datasets, holds 19192 unique experimentally validated therapeutic peptide sequences including 1099 anticancer ones [38]. However, using Blast search on whole this database with $E$-value = 1 applied for 14 putative bacteriocins of our interest, no hits were found, with an exception of p3seq24 from Clostridium hathewayi DSM 13479. The p3seq24 has $37 \%$ identities $(E$-value $=0.002)$ with Clostridium perfringens UviB (Bactibase ID: BAC090), which is predicted as an antimicrobial and antibacterial bacteriocin. This indicates that all our putative bacteriocins are novel to previously experimentally validated therapeutic peptides.

\subsection{Docking Potential Anticancer Bacteriocins against Cancer} Target p53 DNA Binding Domain. Molecular docking along with atomic force microscopy studies has recently revealed the binding interface of 228 and the DNA-binding domain (DBD) of p53 [39]. The authors suggested that the L1 loop (aa 112-124), a region within the S7-S8 loop (aa 214-236) and Thr 140, Pro 142, Gln 144, Trp 146, Arg 282, and Leu 289 of the p53 $\mathrm{DBD}$ are potential sites for $\mathrm{p} 28$ binding.

We performed molecular docking of p53 DBD (PDB code 1TUP chain B) with p28-azurin using Cluspro server [30]. Cluspro carried out a cluster analysis and the best one had more cluster members and lowest energy compared to other members. We selected the first cluster for validation and further analysis (Figure 2(a)). We have found very similar binding sites which are L1 loop (aa 104, 109-116, 123-134), an amino acid within S7-S8 loop (aa 228), aa 141-148, 268, 282, and 286 (Figure S3, Supplementary Material). Considering that we carried out docking without any prior knowledge of binding site of this complex, these results show a striking overlap between our predicted binding site and that of the authors [39].

One should note that the conformation of a peptide in solution could be different from that observed when it is embedded in the protein. Indeed, the p28-azurin reported in [39] was subjected to molecular dynamics simulation in aqueous solution before docking. The final conformation from molecular dynamics simulation is slightly different from the conformation used in this study, mainly at two terminals of the peptide. However, as mentioned above, our prediction of binding interface between p 28 and p53 DBD is in good agreement with their prediction. This suggests that the configuration of p28 observed in azurin is likely stable in aqueous solution.

Nevertheless, due to the computational cost, performing molecular dynamics simulation may not be able to address all the possible conformations of a peptide. In addition, peptide 
configuration might be changed upon binding to the receptor. Thus one could do peptide flexible docking that searches for the binding interface of the complex while allowing for full flexibility of the peptide. However, this approach still remains a computational challenge owing to the high torsional flexibility of peptides. Recent benchmark [40] has shown that flexible docking of a peptide of up to four amino acids length can be reproduced quite accurately. However, when a peptide has more than four amino acids, the accuracy of prediction declined dramatically [40]. Another benchmark for short peptides (smaller than five) has shown that peptide rigid docking is even more successful than peptide flexible docking [41]. Because all the bacteriocins of interest in this study have more than 30 amino acids, a well-tested program that is able to predict the binding pose of protein-peptide complexes such as Cluspro [30] is therefore a suitable choice.

We also carried out molecular docking of p53 DBD with full-length azurin (PDB code $3 \mathrm{U} 25$ chain A) for validation of the method with the whole protein (Figure 2(b)). The binding interface includes L1 loop (aa 113-124), a region within the S7-S8 loop (aa 224-235), 140, 144, 146, 198, and 199 (Figure S4, Supplementary Material). This binding interface highly overlaps with previous studies as well as the binding interface predicted for $\mathrm{p} 28$. Importantly, the binding region of azurin includes the fragment of p28 (aa 56-72) in which the residue Met 64 is found to bind with p53 DBD Trp 146. Interestingly, even the binding poses of $\mathrm{p} 28$ alone and $\mathrm{p} 28$ within azurin do not exactly overlap, the residue Met 64 in both models binds to the same residue Trp 146 of p53 (Figure 2). In total, we found in our models 8 amino acids of $\mathrm{p} 53$ that bind with both azurin and p28-azurin. They include Phe 113, Leu 114, His 115, Ser 116, Cys 124, Gln 144, Trp146, and Asp 228. These results agree well with recent study of p28 in complex with p53 [39] in which these 8 residues are all found in the binding region.

We have performed molecular docking for all models predicted from previous step using Cluspro [30]. For each complex, we select the first cluster for further analysis. To identify the bacteriocins with anticancer property like azurin or p28-azurin, we assume that they should bind in the same region of p53 as that of azurin or p28-azurin. We found 8 bacteriocins which bind in the same region as p28 and azurin (Table 2). They include p1seq09, p1seq16, p2seq05, p2seq08, $\mathrm{p} 2$ seq20, p3seq02, p3seq17, and p3seq24. Their binding poses are shown in Figure 3. Details of amino acid of p53 DBD involved in binding interface with each bacteriocin are listed in Table S3 (Supplementary Material).

In our previous study, we identified 14 final putative bacteriocins based on the qualitative assessment of the functional properties similar to azurin and laz (lipidated azurin produced by Neisseria meningitides) [19]. In the present work, 8 final candidates were identified based on a system of quantitative analysis of the azurin and p28-like functional prediction scoring with hydrophobicity added, structural modeling, and molecular docking studies against p53. Only three final bacteriocins (p1seq16, p2seq20, and p3seq24) were shared by both two approaches, and interestingly, five novel putative bacteriocins (p1seq09, p2seq05, p2seq08, p3seq02, and $\mathrm{p} 3$ seq17) with potential anticancer activity were discovered in the present study, indicating the similar and improved results from the quantitative and structure-based analyses.

Similarly, using bioinformatics approach, the entire genome of a human commensal bacterium Lactobacillus salivarius was scanned for putative bacteriocins and potentially anticancer bacteriocins were screened through structure prediction and docking studies against the common cancer targets p53, Rb1, and AR with azurin as control. The results have revealed that Lsl_0510 possessed highest binding affinity toward all the three receptors [42]. In the present work, for the first time, a multi-genome-scale screening, homology modeling, and molecular docking study of putative bacteriocins from all 66 dominant bacteria species in human gut microbiome was performed to finally identify 8 candidate peptide drugs with azurin-like anticancer activity. Further in vitro tests were required to make them be ideal candidates for future cancer therapeutics.

In order to develop a cancer therapeutic drug, at least four important properties should be considered: (i) nontoxicity for long term use, (ii) inhibiting and killing any preformed tumor cells, (iii) preventing oncogenic transformation of normal cells to cancer cells, and (iv) being taken orally and not through intravenous injections. The three former properties are shared by $\mathrm{p} 28$ and probably by azurin, although azurin's toxicity and side effects in humans have not yet been assessed [1]. For the fourth feature, p28 is now given intravenously but future technological advances might overcome this problem.

In the present study, 6 of 8 final candidate bacteriocins are small peptides composed of 31 to 77 amino acids (Table 2). These peptides can be administered orally, through subcutaneous or intravenous injections, or even by inhalation. Actually, peptides that have entered the global market are composed of up to 40 or more amino acids. There are only about 11 peptides approved by FDA from 1985 to 2013 and valued more than US\$ 1.0 billion in global sales, which include Copaxone, Lupron, Zoladex, Sandostatin, Lucinactant, Peginesatide, Pasireotide, Carfilzomib, Linaclotide, Teduglutide, and Lixisenatide [1]. However, only a few such as Carfilzomib are indicated for cancer therapy. Thus, along with positive results of p28-azurin in phase I clinical trials in Chicago with 15 stage IV cancer patients [43], small peptides with azurin-like anticancer activity may have promisingly opening potential in cancer therapy in the future.

The remaining two of 8 final candidate bacteriocins are larger proteins composed of 212 to 262 amino acids (Table 2). These proteins have additional domains in their structure and thus may have other cancer growth inhibitory activities that a small peptide lacks. For example, compared to p28, azurin expressed the multidomain and multivalent action to preferentially enter cancer cells and interfere in multiple steps in cancer growth, both intracellular and extracellular [1]. Azurin also have multibiological activity such as antiparasite antiviral activity, including its ability to combat AIDS [14, 15]. Even azurin has been overproduced in Escherichia coli Nissle 1917 probiotic cells to allow the regression of melanoma and breast tumor in the mouse model [44]. Although azurin must often be administrated through intravenous injections rather than orally, it can be chemically synthesized at a modest cost or chemically modified (e.g., insulin can be 


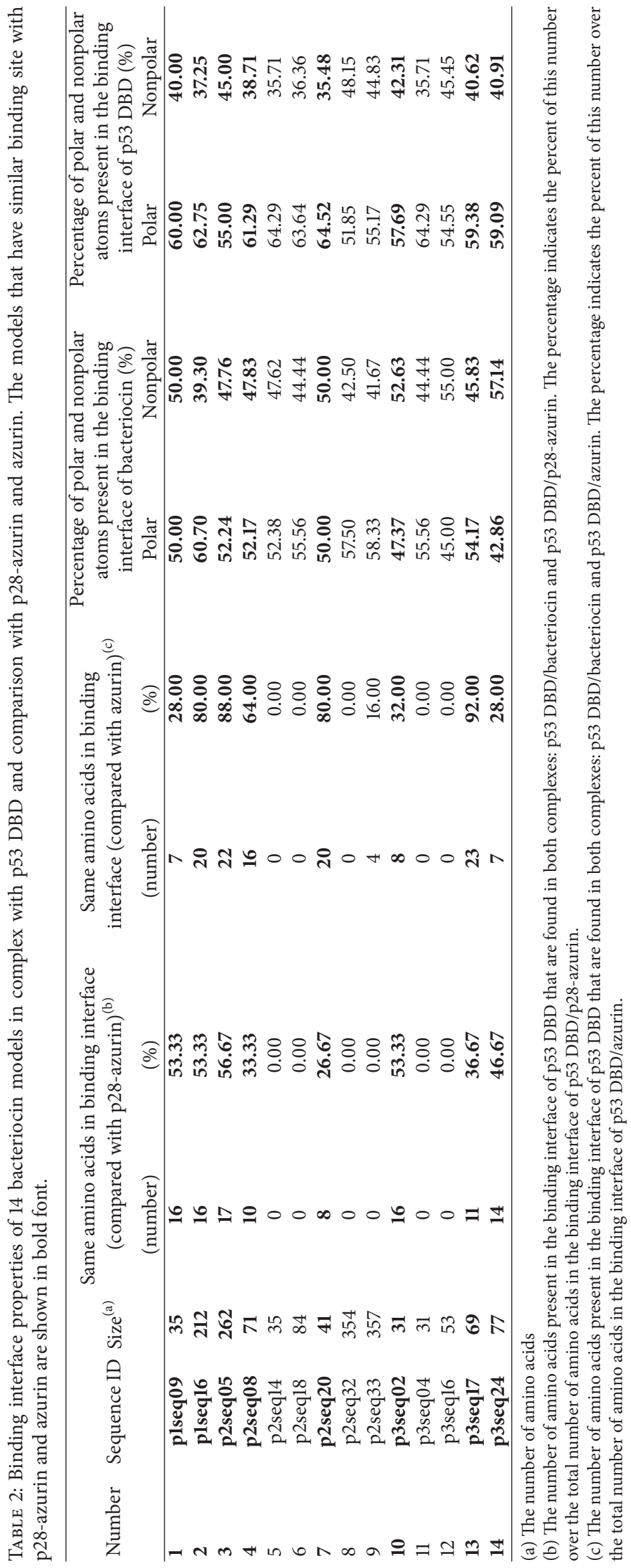




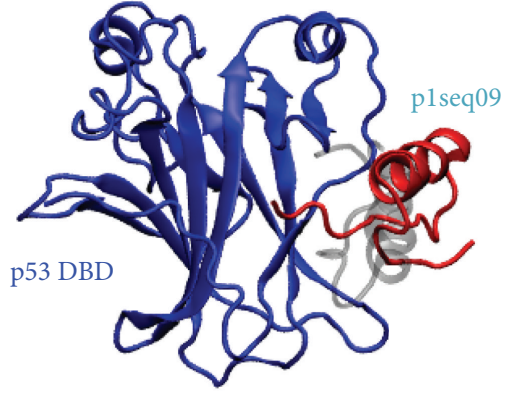

(a)

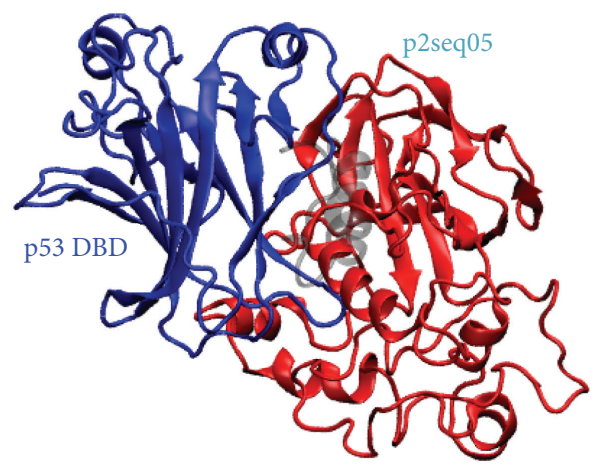

(c)

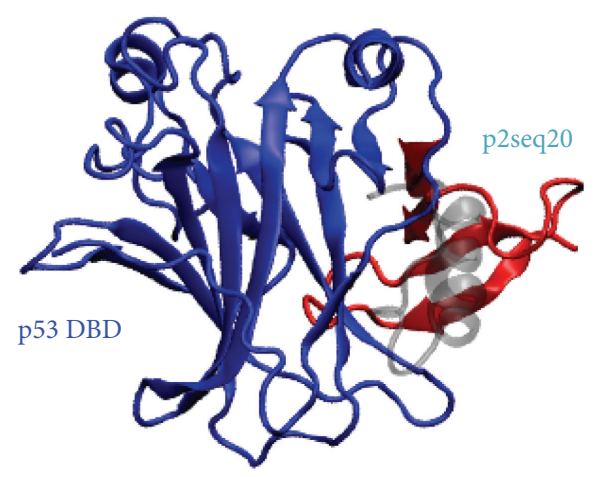

(e)

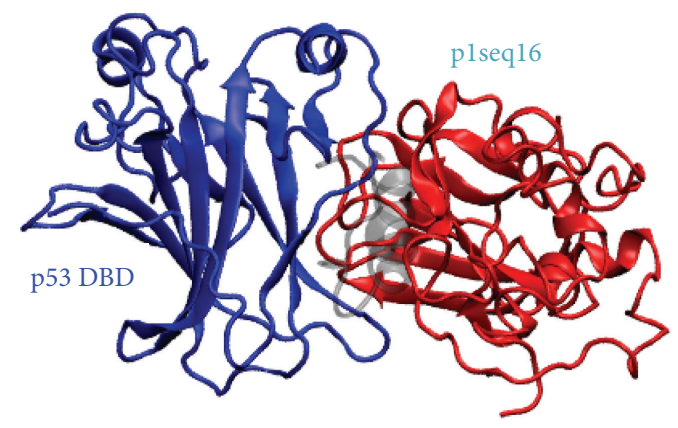

(b)

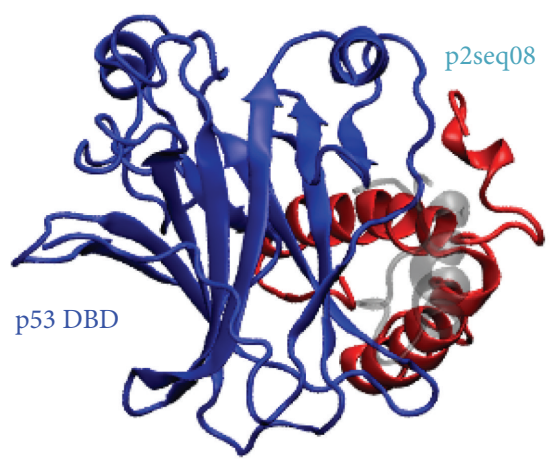

(d)

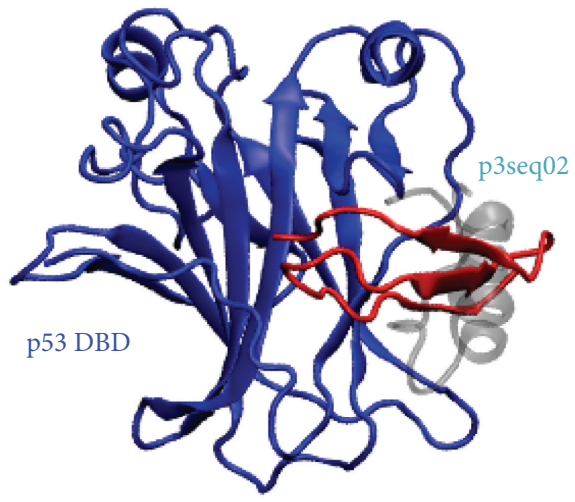

(f)

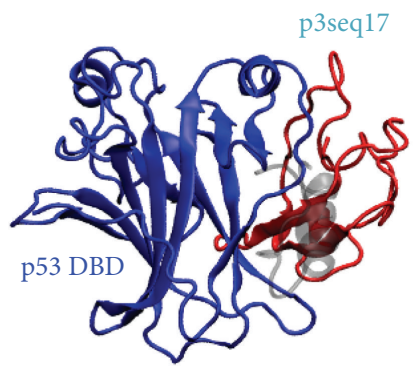

(g)

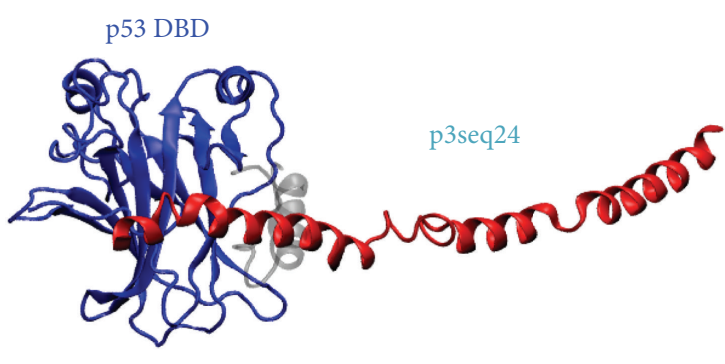

(h)

FIGURE 3: Three-dimensional docking models of 8 bacteriocins that have the highest similar binding site with p28-azurin and azurin when they bind to 53 DBD. The bacteriocins structure is shown in red, p53 DBD in blue, and p28-azurin in grey for reference. 
covalently linked to polyethylene glycol) to become more stable to stomach acids, easily absorbed through gut tract, stable in serum, and less immunogenic [1]. Therefore, large proteins like azurin have more domains in addition to smaller peptides like p28 that can make them a much more effective drug if their efficacy, lack of toxicity, lack of susceptibility to resistance development, and an improvement in mode of administration toward technological advances can be demonstrated in preclinical and human clinical trials.

\section{Conclusion}

Using bioinformatics approaches including functional prediction (scoring), hydrophobic percentage calculation, structural modeling, and molecular docking, at least 8 putative bacteriocins from human gut pathogenic and commensal bacteria have been found to possess functional properties very similar to those of azurin and $\mathrm{p} 28$-azurin with potential anticancer activities. Among them, 3 peptides (p1seq16, p2seq20, and p3seq24) have been shown from our previous study and 5 novel ones (p1seq09, p2seq05, p2seq08, p3seq02, and $\mathrm{p} 3 \mathrm{seq} 17$ ) are discovered for the first time here. The results herald a new era of drug development and contribute to better human health.

If the pathogenic and commensal bacteria with long term residence in human body produce these proteins to defend their habitat from invaders such as cancers and other deadly diseases, this can lead us to identify the novel anticancer drugs from human microflora. The discovery of these drugs has just been started.

\section{Disclosure}

Chuong Nguyen is the first author.

\section{Competing Interests}

The authors declare that there is no conflict of interests regarding the publication of this paper.

\section{Acknowledgments}

The research was funded by Vietnam National Foundation for Science and Technology Development (NAFOSTED) under Grant no. 106-YS.04-2014.40 to V. D. Nguyen. The authors would like to thank Nguyen Quy Truong for providing code to perform hydrophobic percentage calculation and Tran Phuoc Duy for useful discussion as well as revising the paper.

\section{References}

[1] A. M. Chakrabarty, N. Bernardes, and A. M. Fialho, "Bacterial proteins and peptides in cancer therapy: today and tomorrow," Bioengineered, vol. 5, no. 4, pp. 234-242, 2014.

[2] B. S. Avner, A. M. Fialho, and A. M. Chakrabarty, "Overcoming drug resistance in multi-drug resistant cancers and microorganisms: a conceptual framework," Bioengineered, vol. 3, no. 5, pp. 262-270, 2012.
[3] S. Felgner, D. Kocijancic, M. Frahm, and S. Weiss, "Bacteria in cancer therapy: renaissance of an old concept," International Journal of Microbiology, vol. 2016, Article ID 8451728, 14 pages, 2016.

[4] W. B. Coley, "II. Contribution to the knowledge of sarcoma," Annals of Surgery, vol. 14, no. 3, pp. 199-220, 1891.

[5] M. Riley, "Bacteriocins, biology, ecology, and evolution," in Encyclopedia of Microbiology, pp. 32-44, Elsevier, New York, NY, USA, 2009.

[6] G. Cornut, C. Fortin, and D. Soulières, "Antineoplastic properties of bacteriocins: revisiting potential active agents," American Journal of Clinical Oncology, vol. 31, no. 4, pp. 399-404, 2008.

[7] T. Yamada, M. Goto, V. Punj et al., "Bacterial redox protein azurin, tumor suppressor protein p53, and regression of cancer," Proceedings of the National Academy of Sciences of the United States of America, vol. 99, no. 22, pp. 14098-14103, 2002.

[8] T. Yamada, Y. Hiraoka, M. Ikehata et al., "Apoptosis or growth arrest: modulation of tumor suppressor p53's specificity by bacterial redox protein azurin," Proceedings of the National Academy of Sciences of the United States of America, vol. 101, no. 14, pp. 4770-4775, 2004.

[9] T. Yamada, A. M. Fialho, V. Punj, L. Bratescu, T. K. Das Gupta, and A. M. Chakrabarty, "Internalization of bacterial redox protein azurin in mammalian cells: entry domain and specificity," Cellular Microbiology, vol. 7, no. 10, pp. 1418-1431, 2005.

[10] L. Jia, G. S. Gorman, L. U. Coward et al., "Preclinical pharmacokinetics, metabolism, and toxicity of azurin-p28 (NSC745104) a peptide inhibitor of p53 ubiquitination," Cancer Chemotherapy and Pharmacology, vol. 68, no. 2, pp. 513-524, 2011.

[11] V. De Grandis, A. R. Bizzarri, and S. Cannistraro, "Docking study and free energy simulation of the complex between p53 DNA-binding domain and azurin," Journal of Molecular Recognition, vol. 20, no. 4, pp. 215-226, 2007.

[12] M. Taranta, A. R. Bizzarri, and S. Cannistraro, "Probing the interaction between $\mathrm{p} 53$ and the bacterial protein azurin by single molecule force spectroscopy," Journal of Molecular Recognition, vol. 21, no. 1, pp. 63-70, 2008.

[13] M. Taranta, A. R. Bizzarri, and S. Cannistraro, "Modeling the interaction between the N-terminal domain of the tumor suppressor p53 and azurin," Journal of Molecular Recognition, vol. 22, no. 3, pp. 215-222, 2009.

[14] A. Chaudhari, A. M. Fialho, D. Ratner et al., "Azurin, Plasmodium falciparum malaria and HIV/AIDS: inhibition of parasitic and viral growth by azurin," Cell Cycle, vol. 5, no. 15, pp. 1642$1648,2006$.

[15] A. Naguleswaran, A. M. Fialho, A. Chaudhari, C. S. Hong, A. M. Chakrabarty, and W. J. Sullivan Jr., "Azurin-like protein blocks invasion of Toxoplasma gondii through potential interactions with parasite surface antigen SAG1," Antimicrobial Agents and Chemotherapy, vol. 52, no. 2, pp. 402-408, 2008.

[16] A. M. Fialho, N. Bernardes, and A. M. Chakrabarty, "Recent patents on live bacteria and their products as potential anticancer agents," Recent Patents on Anti-Cancer Drug Discovery, vol. 7, no. 1, pp. 31-55, 2012.

[17] J.-H. Cho, M.-H. Lee, Y.-J. Cho, B.-S. Park, S. Kim, and G.-C. Kim, "The bacterial protein azurin enhances sensitivity of oral squamous carcinoma cells to anticancer drugs," Yonsei Medical Journal, vol. 52, no. 5, pp. 773-778, 2011.

[18] C. Hetz, M. R. Bono, L. F. Barros, and R. Lagos, "Microcin E492, a channel-forming bacteriocin from Klebsiella pneumoniae, 
induces apoptosis in some human cell lines," Proceedings of the National Academy of Sciences of the United States of America, vol. 99, no. 5, pp. 2696-2701, 2002.

[19] V. D. Nguyen and H. H. C. Nguyen, "Molecular screening of azurin-like anticancer bacteriocins from human gut microflora using bioinformatics," in Advances in Intelligent Systems and Computing, pp. 219-229, Springer, Berlin, Germany, 2015.

[20] S. Schloissnig, M. Arumugam, S. Sunagawa et al., "Genomic variation landscape of the human gut microbiome," Nature, vol. 493, no. 7430, pp. 45-50, 2013.

[21] A. J. van Heel, A. de Jong, M. Montalbán-López, J. Kok, and O. P. Kuipers, "BAGEL3: automated identification of genes encoding bacteriocins and (non-)bactericidal posttranslationally modified peptides," Nucleic Acids Research, vol. 41, pp. W448-W453, 2013.

[22] L. J. Jensen, R. Gupta, N. Blom et al., "Prediction of human protein function from post-translational modifications and localization features," Journal of Molecular Biology, vol. 319, no. 5, pp. 1257-1265, 2002.

[23] L. J. Jensen, R. Gupta, H.-H. Stærfeldt, and S. Brunak, "Prediction of human protein function according to Gene Ontology categories," Bioinformatics, vol. 19, no. 5, pp. 635-642, 2003.

[24] T. M. Karpiński and A. K. Szkaradkiewicz, "Anticancer peptides from bacteria," Bangladesh Journal of Pharmacology, vol. 8, no. 3, pp. 343-348, 2013.

[25] J. Yang and Y. Zhang, "I-TASSER server: new development for protein structure and function predictions," Nucleic Acids Research, vol. 43, no. 1, pp. W174-W181, 2015.

[26] J. Ko, H. Park, L. Heo, and C. Seok, "GalaxyWEB server for protein structure prediction and refinement," Nucleic Acids Research, vol. 40, no. 1, pp. W294-W297, 2012.

[27] D. Xu and Y. Zhang, "Improving the physical realism and structural accuracy of protein models by a two-step atomiclevel energy minimization," Biophysical Journal, vol. 101, no. 10, pp. 2525-2534, 2011.

[28] V. B. Chen, W. B. Arendall III, J. J. Headd et al., "MolProbity: allatom structure validation for macromolecular crystallography," Acta Crystallographica, Section D: Biological Crystallography, vol. 66, no. 1, pp. 12-21, 2010.

[29] A. C. Joerger, H. C. Ang, and A. R. Fersht, "Structural basis for understanding oncogenic p53 mutations and designing rescue drugs," Proceedings of the National Academy of Sciences of the United States of America, vol. 103, no. 41, pp. 15056-15061, 2006.

[30] S. R. Comeau, D. W. Gatchell, S. Vajda, and C. J. Camacho, "ClusPro: an automated docking and discrimination method for the prediction of protein complexes," Bioinformatics, vol. 20, no. 1, pp. 45-50, 2004.

[31] D. J. Naisbitt, S. F. Gordon, M. Pirmohamed, and B. K. Park, "Immunological principles of adverse drug reactions: the initiation and propagation of immune responses elicited by drug treatment," Drug Safety, vol. 23, no. 6, pp. 483-507, 2000.

[32] D. G. Lee, K.-S. Hahm, Y. Park et al., "Functional and structural characteristics of anticancer peptide Pep27 analogues," Cancer Cell International, vol. 5, article 21, 2005.

[33] Y.-B. Huang, X.-F. Wang, H.-Y. Wang, Y. Liu, and Y. Chen, "Studies on mechanism of action of anticancer peptides by modulation of hydrophobicity within a defined structural framework," Molecular Cancer Therapeutics, vol. 10, no. 3, pp. 416-426, 2011.

[34] A. M. Chakrabarty, "Bacterial proteins: a new class of cancer therapeutics," Journal of Commercial Biotechnology, vol. 18, no. $1,2012$.
[35] A. Bolhassani, "Potential efficacy of cell-penetrating peptides for nucleic acid and drug delivery in cancer," Biochimica et Biophysica Acta, vol. 1816, no. 2, pp. 232-246, 2011.

[36] A. Suau, R. Bonnet, M. Sutren et al., "Direct analysis of genes encoding 16S rRNA from complex communities reveals many novel molecular species within the human gut," Applied and Environmental Microbiology, vol. 65, no. 11, pp. 4799-4807, 1999.

[37] S. Wu, J. Skolnick, and Y. Zhang, "Ab initio modeling of small proteins by iterative TASSER simulations," BMC Biology, vol. 5, article 17, 2007.

[38] S. Singh, K. Chaudhary, S. K. Dhanda et al., "SATPdb: a database of structurally annotated therapeutic peptides," Nucleic Acids Research, vol. 44, no. 1, pp. D1119-D1126, 2016.

[39] T. Yamada, K. Christov, A. Shilkaitis et al., "P28, A first in class peptide inhibitor of cop1 binding to p53," British Journal of Cancer, vol. 108, no. 12, pp. 2495-2504, 2013.

[40] A. S. Hauser and B. Windshügel, "LEADS-PEP: a benchmark data set for assessment of peptide docking performance," Journal of Chemical Information and Modeling, vol. 56, no. 1, pp. 188-200, 2016.

[41] R. Rentzsch and B. Y. Renard, "Docking small peptides remains a great challenge: an assessment using AutoDock Vina," Briefings in Bioinformatics, vol. 16, no. 6, pp. 1045-1056, 2015.

[42] F. Shaikh, P. Abhinand, and P. Ragunath, "Identification \& Characterization of lactobacillus salavarius bacteriocins and its relevance in cancer therapeutics," Bioinformation, vol. 8, no. 13, pp. 589-594, 2012.

[43] M. A. Warso, J. M. Richards, D. Mehta et al., "A first-inclass, first-in-human, phase I trial of p28, a non-HDM2mediated peptide inhibitor of p53 ubiquitination in patients with advanced solid tumors," British Journal of Cancer, vol. 108, no. 5, pp. 1061-1070, 2013.

[44] Y. Zhang, Y. Zhang, L. Xia et al., "Escherichia coli Nissle 1917 targets and restrains mouse b16 melanoma and $4 \mathrm{~T} 1$ breast tumors through expression of azurin protein," Applied and Environmental Microbiology, vol. 78, no. 21, pp. 7603-7610, 2012. 

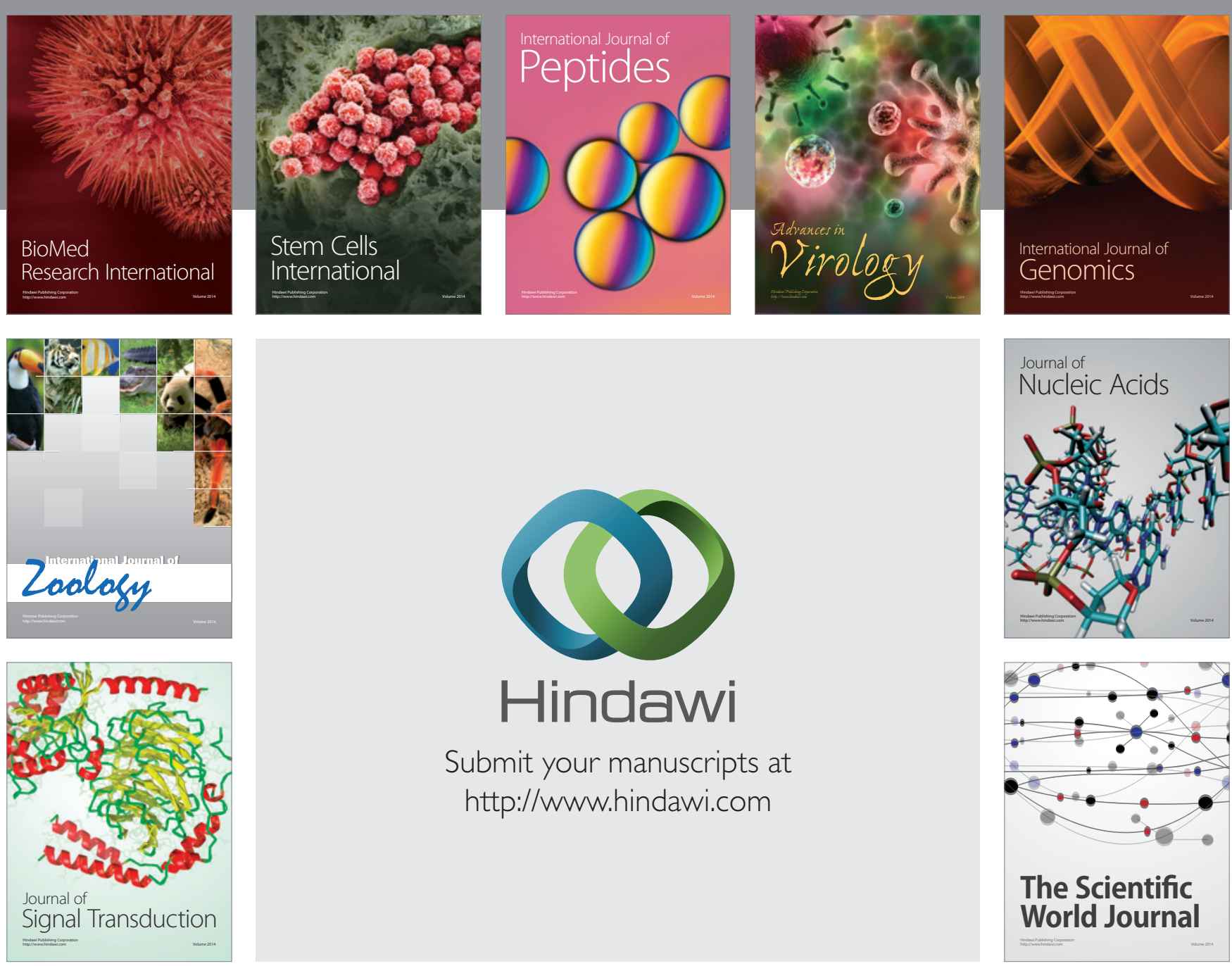

Submit your manuscripts at

http://www.hindawi.com
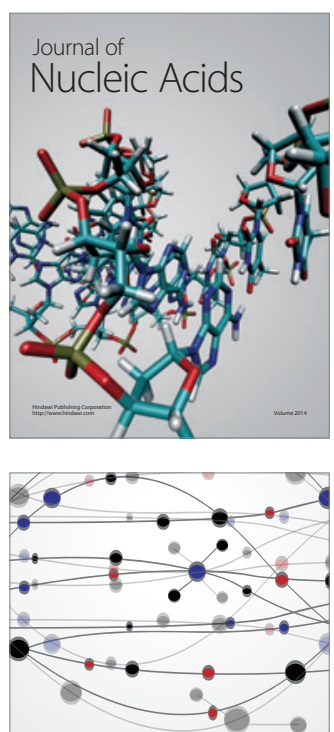

The Scientific World Journal
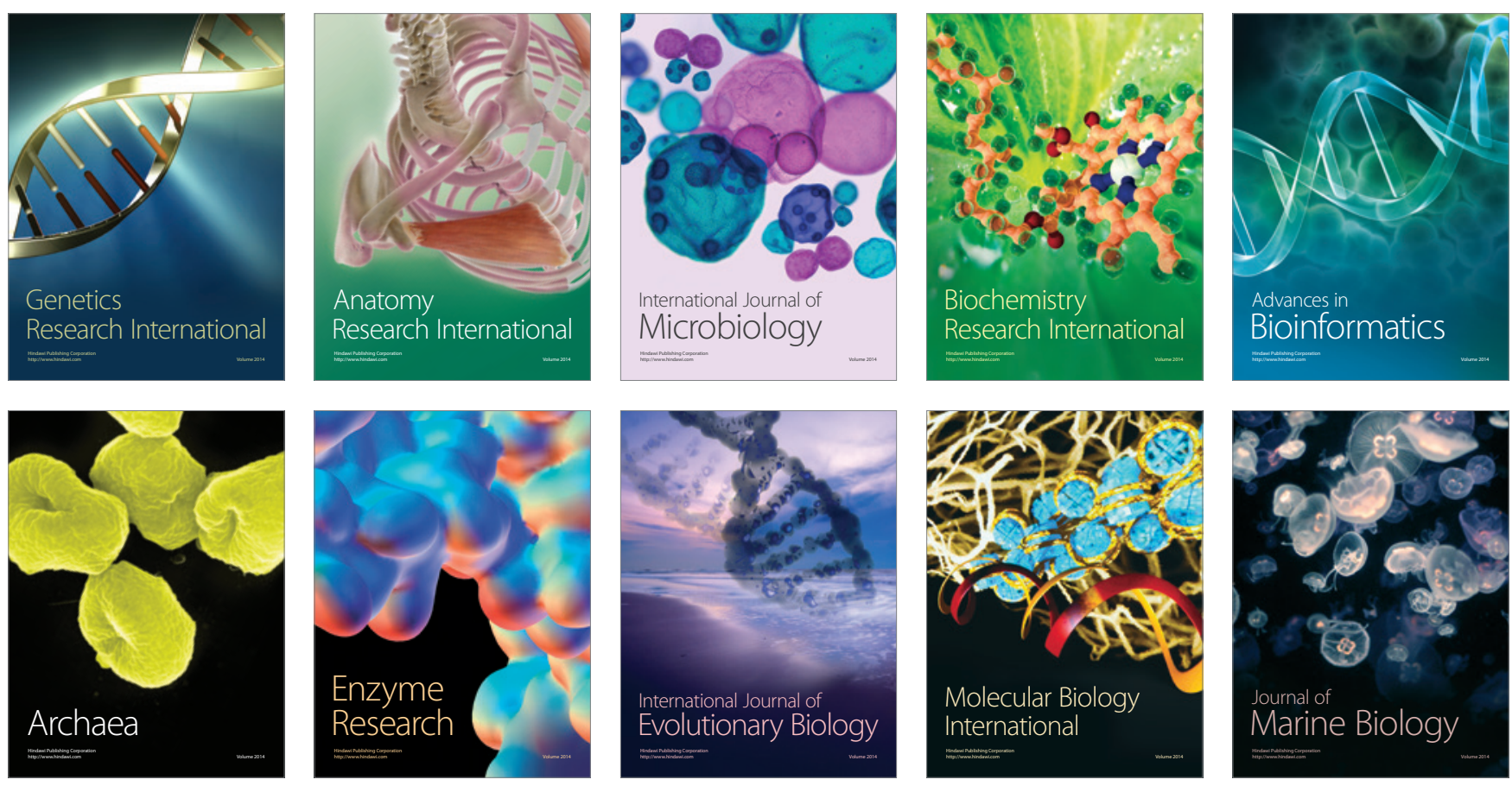